\title{
Endobronchial ultrasound in Dieulafoy's disease of the bronchus: an additional application of EBUS
}

\author{
C. Gurioli, G.L. Casoni, C. Gurioli, S. Tomassetti, M. Romagnoli, \\ C. Ravaglia, V. Poletti
}

ABSTRACT: Endobronchial ultrasound in Dieulafoy's disease of the bronchus: an additional application of EBUS. C. Gurioli, G.L. Casoni, C. Gurioli, S. Tomassetti, M. Romagnoli, C. Ravaglia, V. Poletti.

Dieulafoy's disease is a rare vascular malformation represented by an abnormally enlarged submucosal arterial vessel. This malformation is mostly found in gastrointestinal tract causing spontaneous bleeding although a few cases have been described in the bronchial tree. Recognizing Dieulafoy's malformation is crucial for the bronchoscopist in order to avoid biopsy that can lead to a massive hemoptysis, sometimes fatal. In this case report we show the clinical utility of endobronchial ultrasound (EBUS) in the evaluation of bronchial alteration suspicious for Dieulafoy's malformation.

Monaldi Arch Chest Dis 2010; 73: 4, 166-168.

Keywords: Dieulafoy's disease, Endobronchial ultrasound, Hemoptysis.

Interventional Pulmonology Unit - Department of Thoracic Diseases, P.L. Pierantoni - G.B. Morgagni Hospital, Via Carlo Forlanini 34, 47100, Forli, Italy.

Correspondence: Venerino Poletti MD, venerino.poletti@gmail.com

\section{Introduction}

Dieulafoy's disease takes its name from a French surgeon who, in 1898, first described the erosion of a dysplastic artery underlying the gastric mucosa in three cases of massive gastrointestinal bleeding [1].

This lesion mainly involves the gastrointestinal tract, but it can also affect the respiratory tract, causing even life-threatening hemoptysis due to spontaneous or iatrogenic rupture. The bronchoscopist should be aware of this rare malformation in order to avoid bronchial biopsies that can lead to a life-threatening hemoptysis.

Endobronchial ultrasound (EBUS) is a well-known technique for the evaluation of mediastinal structures and pulmonary parenchymal lesions with the possibility of rising the diagnostic yield of bioptic manoeuvres. The Doppler examination enables the bronchoscopist to differentiate better the arterial and venous vessels from solid structures as lymphnodes or neoplastic lesions [2]. This case report show the clinical utility of EBUS in the evaluation of bronchial alteration suspicious for Dieulafoy's malformation.

\section{Case report}

A 65-year-old male, former smoker, with history of asthma and previous kid- ney and prostatic cancer referred to our center for accessional dyspnea. First line assessment did not reveal any abnormalities. He performed high resolution CT scan showing bilateral micronodules. Then he underwent fibrobronchoscopy with execution of bronchial lavage in the right lower lobar bronchus. A more accurate endoscopic exploration revealed a small protrusion of the mucosa with a white cap in the anterior wall of the intermediate bronchus immediately above the middle lobar bronchus (figure 1).

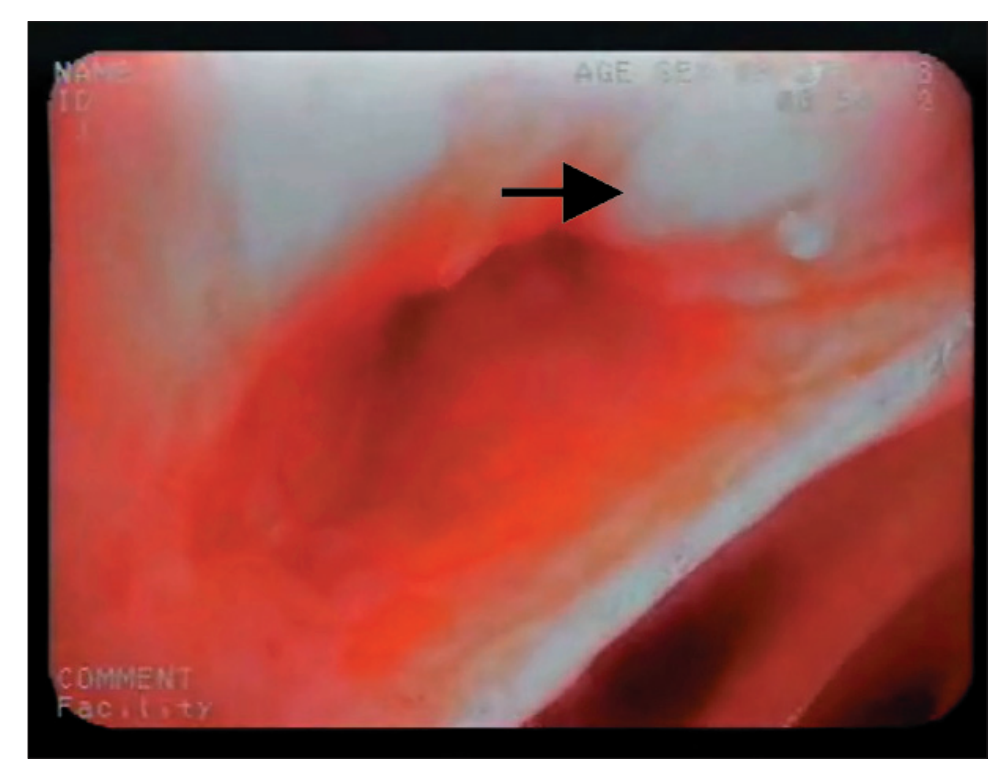

Fig. 1. - Fibrobronchoscopy. A small protrusion with a white cap on the mucosa of the intermediate bronchus immediately above the middle lobar bronchus (arrow). 

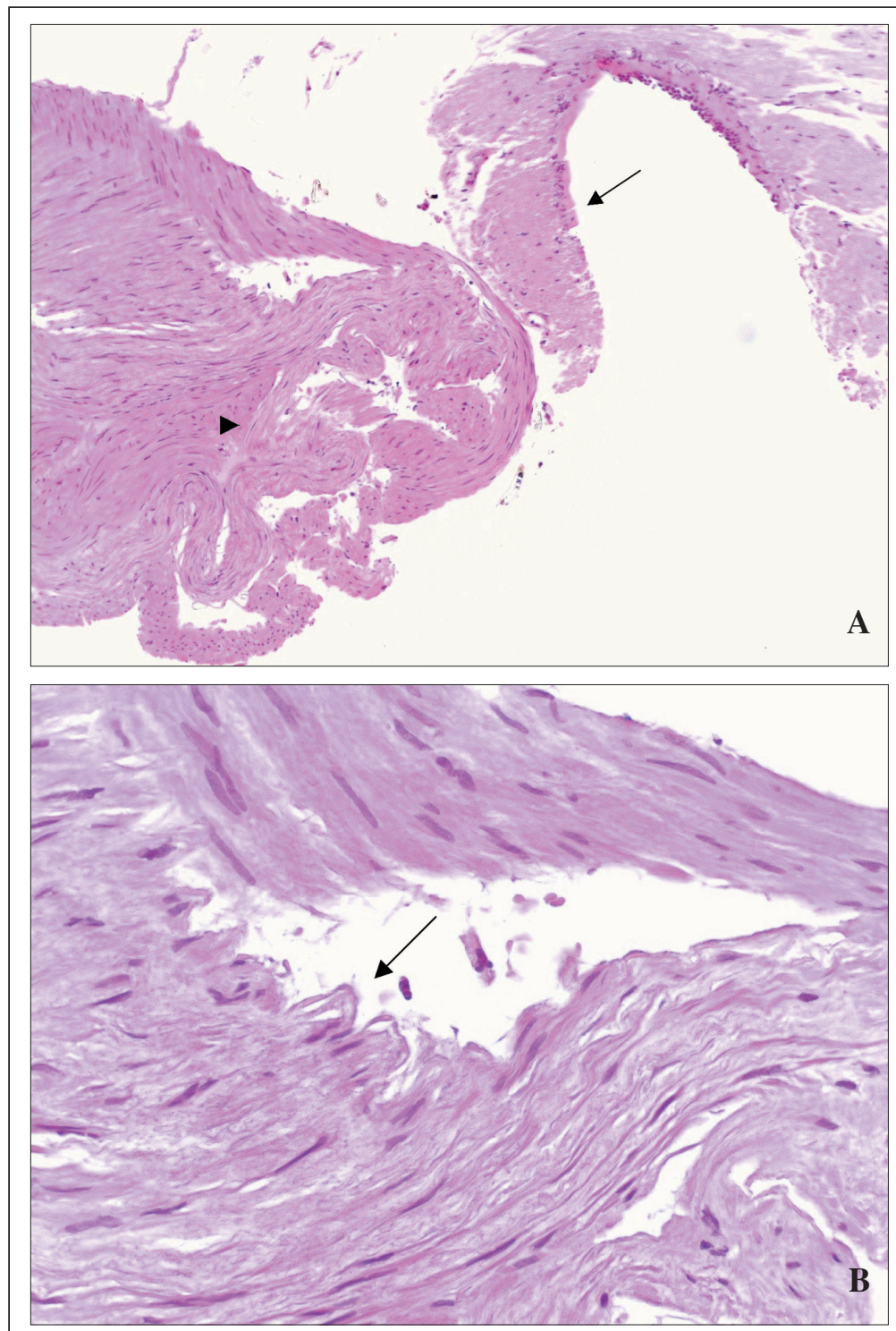

Fig. 2. - Histologic examination, hematoxiline-eosin stain. At magnification 10X a part of wall of little/medium size vessel (arrowhead) can be appreciated under bronchial epithelium (arrow) (A). Magnification 40X allows to identify the elastic lamina, that characterizes the arterial wall. (B)

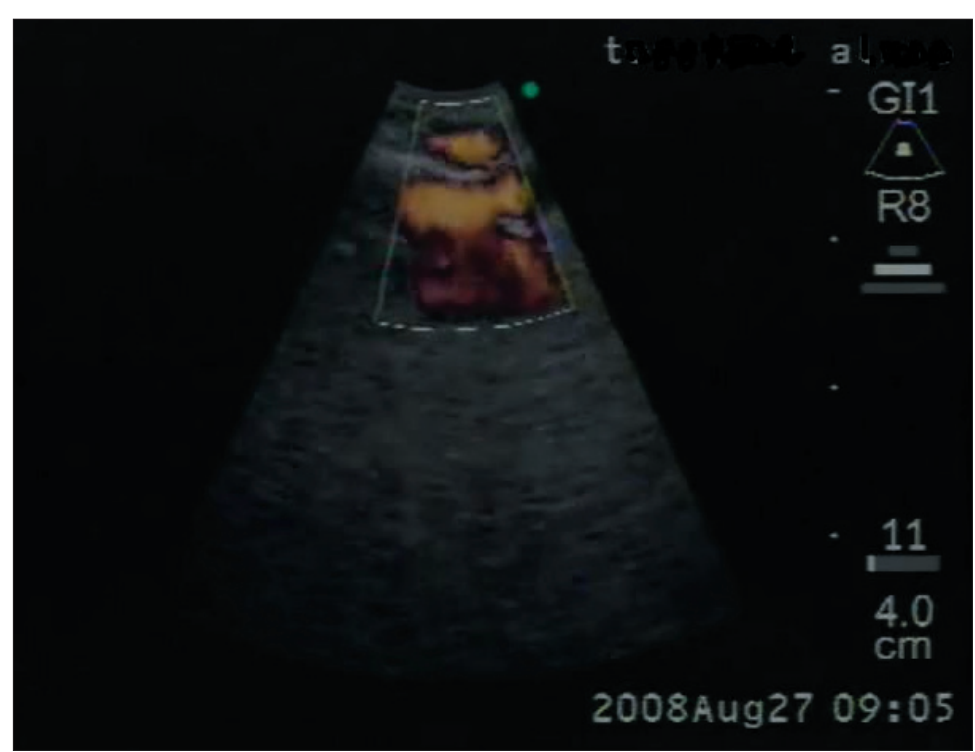

Fig. 3. - Endobronchial ultrasound. Color-Doppler examination performed on the mucosal lesion showed a little vessel (diameter $1.5 \mathrm{~mm}$ ) with arterial flow $1 \mathrm{~mm}$ below the surface.
We performed a biopsy of the lesion that determined conspicuous bleeding resolved after 10 minutes of continuous aspiration and instillation of cold physiologic solution, adrenaline $(0.1 \mathrm{mg}$ repeated twice) and tranexamic acid (total $3000 \mathrm{mg}$ ). The patient did not experience any other complications after the examination.

Histologic evaluation showed a fragment of bronchial mucosa with focal elastosis of subepitelial connective tissue and part of a wall of a small sized arterial vessel (figure 2).

In view of these findings, we performed a subsequent evaluation via rigid bronchoscopy with linear probe 7.5 $\mathrm{MHz}$ endobronchial ultrasound (bronchoscope model BF-UC160FOL8) that showed a small vessel (diameter $1.5 \mathrm{~mm}$ ) with arterial flow at Doppler images, covered by bronchial wall underlying $1 \mathrm{~mm}$ the lesion previously biopsied (figure 3).

\section{Discussion}

Dieulafoy's disease is a rare vascular malformation characterised by a submucosal arterial vessel abnormally enlarged. This malformation is mostly found in gastrointestinal tract causing spontaneous bleeding although some cases it is described in the bronchial tree.

There is some controversy regarding the origin of this malformation in the bronchus. It is not clear yet if it's congenital or acquired. Although most of the authors assign the origin to the systemic arterial tree, the failure of arterial embolisation could argue for the provenance from the pulmonary tree [3].

In the management of a massive hemoptysis from Dieulafoy's disease the surgical resection still remains the main diagnostic and therapeutic approach, as emphasised in most recent case series $[4,5]$.

In general, in cases of haemoptysis bronchoscopy plays an important role in detecting the site of the bleeding and for the treatment of endobronchial bleeding lesions if required.

The identification of Dieulafoy's malformation is crucial for the bronchoscopist in order to avoid biopsies that can lead to a massive hemoptysis, sometimes fatal [6]. Recently Löshhorn et al. described a case of near fatal hemoptysis after biopsy of a bronchial Dieulafoy's malformation [7]. In their report they postulated the usefulness of EBUS in detecting vascular abnormalities in mucosal alteration suspected for Dieulafoy's disease. 
Moreover endoscopic ultrasound (EUS) with color-Doppler examination is already a well-established technique for the identification and management of Dieulafoy's malformation of the gastrointestinal tract [8].

In our case the bronchial biopsy caused considerable unexpected bleeding. The EBUS with Doppler images achieved "a posteriori" allowing easy identification of vascular malformation under mucosal protrusion, in accordance with the histologic finding.

To the best of our knowledge the present report first describes the EBUS examination in the bronchial version of this rare disease underlining the potential role in clinical practice of this novel application.

\section{References}

1. Dieulafoy G. Exulceratio simplex. L'intervention chirurgicale dans les hématémèses foudroyantes consécutives à l'exulceration simple de l'estomac. Bull Acad Méd 1898; 49: 49-84.
2. Casoni GL, Gurioli C, Romagnoli M, Poletti V. Diagnosis of pulmonary thromboembolism with endobronchial ultrasound. Eur Respir J 2008: 32: 1416-7.

3. Pomplun S, Sheaff MT. Dieulafoy's disease of the bronchus: un uncommon entity. Histopathology 2005; 46: 598-599.

4. Gharagozloo F, Douglas R, Margolis M, et al. Dieulafoy's lesion of the bronchus: review of the literature and report of the 13th case. J Bronchol 2008; 15: 38-40.

5. Parrot A, Antoine M, Khalil A, et al. Approach to diagnosis and pathological examination in bronchial Dieulafoy disease: a case series. Respir Res 2008; 9: 58.

6. van der Werf TS, Timmer A, Zijlstra JG. Fatal haemorrhage from Dieulafoy's disease of the bronchus. Thorax 1999; 54: 184-185.

7. Löshhorn C, Nierhoff N, Mayer R, Zaunbauer W, Neuweiler J, Knoblauch A. Dieulafoy's disease of the lung: a potential disaster for the bronchoscopist. Respiration 2006; 73 : 562-565.

8. Folvik G, Nesje LB, Berstad A, Odegaard S. Endosonography-guided endoscopic band ligation of Dieulafoy's malformation. Endoscopy 2001; 33: 636638.

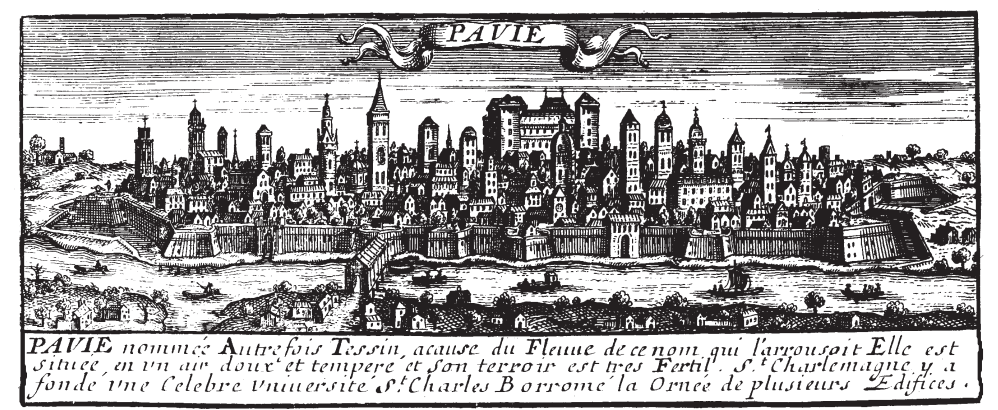

